\title{
THE ATHLETES' BODY SHAPES THE ATHLETES' MIND - NEW PERSPECTIVES ON MENTAL ROTATION PERFORMANCE IN ATHLETES
}

\author{
Thomas Heinen \\ University of Hildesheim, Germany \\ E-mail: thomas.heinen@uni-hildesheim.de \\ Damian Jeraj \\ German Sport University Cologne, Germany \\ E-mail: d.jeraj@dshs-koeln.de
}

\begin{abstract}
Mentally rotating the image of an object is one fundamental cognitive ability in humans. Recent theoretical developments and empirical evidences highlight the potential role of the sensory-motor system, when analysing and understanding mental rotation. Therefore, the purpose of this study was to investigate the role of specific sensory-motor experience on mental rotation performance in gymnasts. $N=40$ male gymnasts with either clockwise or anticlockwise rotation preference in a forward twisting layout salto performed a psychometric mental rotation test with either rotation-preference congruent or rotation-preference incongruent stimuli. Results revealed that choice reaction times differed clearly as a function of Angular Rotation between the stimuli figures. Gymnasts who preferred a clockwise rotation preference showed faster choice reaction times when the rotation direction of the reference figure was clockwise, and vice versa. The results clearly support the notion, that mental rotation performance varies as a function of sensory-motor system characteristics between different people. It is concluded, that sensory-motor experience in a particular sport may facilitate cognitive processing of experience-congruent stimuli. This may be advantageous for situations in which people are engaged in observing sport performance (i.e., judges, coaches). This conclusion could furthermore contribute to the training of athletes from sports such as sky-diving, scuba-diving, and climbing, where losses of spatial orientation can be life-threatening.
\end{abstract}

Key words: functional equivalence hypothesis, gymnastics, rotation preference, sensory-motor experience.

\section{I ntroduction}

Mentally rotating the image of an object is seen as one fundamental cognitive ability in humans (Sternberg, 2003). It has received considerable attention by a great number of researchers from various disciplines (Munzert, Lorey, \& Zentgraf, 2009; Zacks, 2008). New theoretical developments and empirical evidences highlight the potential role of the sensory-motor system in general, and sensory-motor experience in particular, when analysing and understanding perceptual and cognitive processes (Gibbs, 2006; Wexler, Kosslyn, \& Berthoz, 1998). Therefore the purpose of this study 
was to investigate the role of specific sensory-motor experience on mental rotation performance in gymnasts.

In a classical mental rotation task, participants usually observe pairs of pictures showing three-dimensional cuboid-like objects (Shepard \& Metzler, 1971). These objects exhibit different rotation angles and participants are asked to indicate if one object is a rotation of the other object or not. The results usually show a (linear) function between the angular rotation of the two objects and participants' reaction time, supporting the functional-equivalence hypothesis (Jolicoeur \& Kosslyn, 1985; Paivio, 1990). This hypothesis states that the percepts one constructs mentally are not identical, but functionally equivalent to the physical phenomena they represent. Therefore, phenomena that occur in the physical world should have their functional equivalent in their corresponding mental representations. Thus, if one rotates an object in the physical world it would take longer to rotate the object, if it has to be rotated a larger angle.

In light of the functional equivalence hypothesis one may argue that if there is a congruence between the stimulus (or characteristics of it) in a mental rotation task, and the person (or characteristics of it) performing the mental rotation task, then this would facilitate the mental rotation process (Heinen, 2013). Amorim, Isableu and Jarraya (2006) had for instance participants perform a mental rotation test with stimuli of different complexity. Mental rotation performance on classical, cuboid-like figures was for instance compared to mental rotation performance on cuboid-like figures with body characteristics (e.g., adding a head to the cubes), and also to mental rotation performance on computer-generated human-like figures that matched the spatial configuration of the cuboid-like figures. Results revealed that participants exhibited a better mental rotation performance when the stimuli comprised bodily characteristics or were human-like.

From recent theoretical developments and empirical evidence one may argue that the functional equivalence just mentioned is at least in part shaped by the sensory-motor system one possesses, since this system is the basis for interaction with the environment (Barsalou, 2008; Gibbs, 2006). Thus, mental rotation performance is thought to vary as a function of current states of the sensorymotor system. Ionta, Fourkas, Fiorio and Aglioti (2007) investigated for instance the influence of participants' hand posture on mental rotation performance of hands and feet. Results revealed worse mental rotation performance when the hands were held in an unusual posture as compared to an anatomical posture. Lenggenhager, Lopez and Blanke (2008) applied galvanic vestibular stimulation while participants performed a mental rotation test. Results indicated a specific interfering effect of galvanic vestibular stimulation on mental rotation performance, that was modulated by participants' sensory sensation during galvanic vestibular stimulation. The results just described highlight the role of the current state of the sensory-motor system in mental rotation.

Nevertheless, different people possess different sensory-motor system characteristics. Especially athletes have accumulated massive sensory and motor experience over their years of practice in performing different actions and activities (Blake \& Shiffar, 2007; O'Regan \& Noë, 2001). This experience usually goes along with adaptations in the various body systems, so that athletes' sensory-motor system usually considerably differs from non-athletes (Enoka, 2003). As a consequence one would expect that mental rotation performance varies as a function of sensorymotor system characteristics between different people. Pietsch and Jansen (2012) investigated for instance the relationship between physical and musical activity, and mental rotation performance. In particular it was found that music and sport students exhibited faster reaction times in a mental rotation test as compared to education students. The results just described support the notion that mental rotation performance varies as a function of sensory-motor system characteristics between different people. Furthermore, Moreau, Clerc, Mansy-Dannay and Guerrien (2012) had participants complete a mental rotation test before and after a specific physical training in two different sports. One sport comprised mental rotation ability (wrestling) whilst the other sport did not (running). In particular, the results showed that the wrestling group outperformed the running group in the mental rotation test after completing the physical training. The results just described highlight the role that sensory-motor experience may play in mental rotation performance.

Taken together, one may argue that athletes' specific sensory-motor experience could influence mental rotation performance. Following this, the main purpose of this study was to investigate the role of specific sensory-motor experience on mental rotation performance in gymnasts. It was 
predicted that gymnasts' mental rotation performance on gymnast-like stimuli varies as a function of gymnasts' rotation preference when performing forward twisting saltos. In particular, it was expected that gymnasts who prefer a clockwise rotation direction in a forward twisting salto show faster reaction times on rotation-preference congruent stimuli in a mental rotation test as compared to rotation-preference incongruent stimuli (Heinen, 2013). However, since gymnasts were asked to perform a mental rotation test, it was also expected that mental rotation performance in general varies as a function of the angular rotation between the two stimuli figures (Shepard \& Metzler, 1971).

\section{Methodology of Research}

\section{General Background of Research}

Perceptual and cognitive processes seem to be influenced by characteristics of gymnasts' sensory-motor system (Amorim et al., 2006; Gibbs, 2006; Heinen, 2013). The functional-equivalence hypothesis states that the percepts one constructs mentally are functionally equivalent to the physical phenomena they represent. Thus, one may assume that if there is a congruence between the stimulus (or characteristics of it) in a mental rotation task, and the person (or characteristics of it) performing the mental rotation task, then this would facilitate the mental rotation process (Heinen, 2013). In order to evaluate this assumption, a mental rotation paradigm was utilized, with gymnasts as participating subjects.

\section{Sample of Research}

Participants were $N=40$ male gymnasts ( $M_{\text {age }}: 18$ years, $S D=3$ years). Gymnasts had at least $11 \pm 4$ years of experience in artistic gymnastics and practiced on average $10 \pm 3$ hours per week. They reported to have national experience due to participation in national gymnastics championships, and participation in national league competitions. They provided their written informed consent prior to participation in this study.

$N=20$ gymnasts reported to have an anti-clockwise rotation preference about the longitudinal axis when performing forward twisting saltos, whereas the remaining $n=20$ gymnasts of the study sample reported to have a clockwise rotation preference about the longitudinal axis when performing forward twisting saltos.

The gymnasts were informed about the general procedure of the study. They were, however, not informed about the experimental manipulation in order to ensure that they remained naïve to the experimental conditions. Gymnasts indicated that they had neither solved a mental rotation test before, nor were specifically trained in mental rotation. The experiment was carried out in accordance with the ethical guidelines of the local university. It was decided to recruit expert gymnasts because they already possess appropriate sensory-motor experiences in their sport (Blake \& Shiffrar, 2007).

\section{Instruments}

A psychometric mental rotation test with human figures as stimulus material was used to assess mental rotation performance (cf., Amorim et al., 2006; Jansen \& Lehmann, 2013). In particular, gymnasts completed a same-different 3-D shape-matching task. The stimuli figures for the shapematching task were created using the computer software Poser 7 (E-Frontier, 2007). 

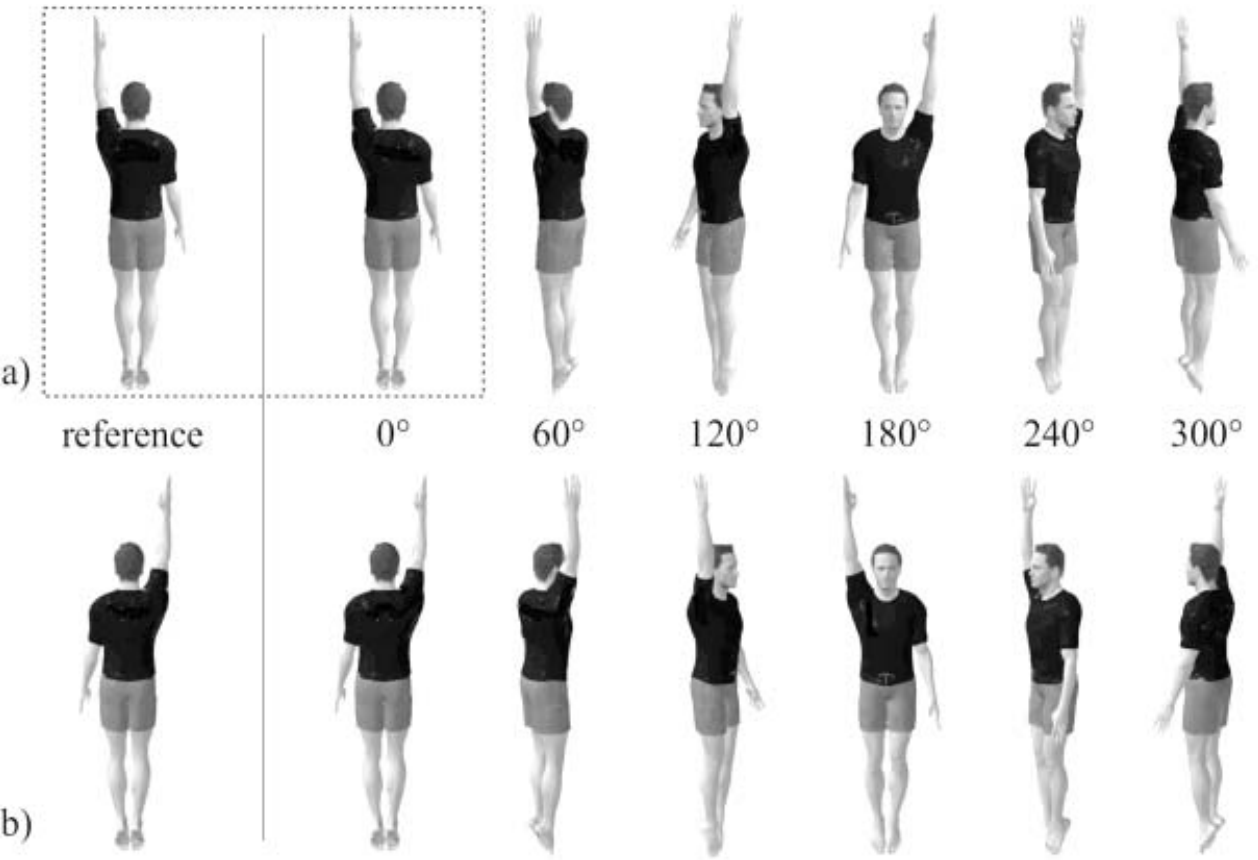

Figure 1: Illustration of the stimuli used in the experiment (congruent condition).
The dotted line represents an exemplary display window during
the experiment. Note: The arm positions shown in the upper part of
the Figure (a) correspond to an anti-clockwise rotation about the
longitudinal axis when performing a forward layout salto (elevating the
left arm and lowering the right arm). The arm positions shown in (b)
correspond to a clockwise rotation about the longitudinal axis during a
forward layout salto (elevating the right arm and lowering the left arm).

Stimuli figures represented a gymnast in an upright posture in front of a white background. One arm was elevated overhead whilst the other arm was lowered towards the floor (see Figure 1). The reference figure was presented from a back perspective, in order help the gymnast matching his perspective to the perspective of the figure (Amorim et al., 2006). Arm positions of the stimuli figures represented different rotation directions about the longitudinal axis in a forward layout twisting salto, which would occur due to the gyroscopic effect of rotating rigid bodies (Yeadon, 1990). According to this effect, an elevation of the left arm together with a lowering of the right arm during a forward layout salto would lead to an anti-clockwise rotation about the longitudinal axis (Figure 1-a), whereas elevating the right arm together with a lowering of the left arm would have the opposite effect (Figure 1-b; Yeadon, 2000). It was decided to manipulate the arm positions of the stimuli, because in particular it was hypothesized that gymnasts' rotation preference would facilitate the mental rotation process of rotation-preference congruent stimuli and vice versa. Since arm positioning is directly related to the rotation of the longitudinal axis when performing a forward layout twisting salto, gymnasts with an anti-clockwise rotation preference should exhibit faster reaction time in a rotation-preference congruent mental rotation task than gymnasts with a clockwise rotation preference.

For each trial, two figures were presented simultaneously on a 20 inch computer monitor (see dotted line in Figure 1). The reference figure was always presented on the left side of the screen. The figure on the right side of the screen was rotated about its longitudinal axis. Rotations ranged from $0^{\circ}$ to $300^{\circ}$ in $60^{\circ}$ increments. Stimuli were presented in either a symmetrical condition or in an asymmetrical condition. In the symmetrical condition, the arm positions of both figures matched. In the asymmetrical condition, the arm positions of both figures were swapped. The gymnasts were instructed to determine as rapidly and accurately as possible whether the two presented figures were same or different using the "Ä" or "A" key, respectively. The keyboard layout was german. The keys were highlighted so that key " $\mathrm{A}$ " could be pressed with the right finger, representing a same response and key " $A$ " could be pressed with the left finger, representing a different response. 
Gymnasts' choice reaction time for each trial was collected by an IBM compatible personal computer using CMC, a software package that can be used to develop psychological experiments (Heinen, 2010). Each gymnast completed 144 trials: 2 (Rotation Direction of Reference Figure: anticlockwise vs. clockwise $) \times 2$ (Arm Position of Figures: symmetrical vs. asymmetrical) $\times 6$ (Angular Rotation of both Figures: $\left.0^{\circ}, 60^{\circ}, 120^{\circ}, 180^{\circ}, 240^{\circ}, 300^{\circ}\right) \times 6$ repetitions. It was decided to repeat each trial six times in order to get a better estimation of the mean choice reaction times for each gymnast (Tabachnick \& Fidell, 2007). Only correct trials were used for further data analysis.

\section{Procedure}

The study was conducted in three phases. All gymnasts were tested individually. In the first phase, the gymnast arrived at the lab and completed the informed consent form. He was briefed about the general purpose and the procedure of the study. The gymnast was, however, left naïve about the experimental manipulation realized in this study. The gymnast was seated in front of the computer monitor and was given 12 practice trials in the mental rotation task in order to familiarize himself with the computer equipment. In the second phase, the gymnast was asked to perform the mental rotation task as described above with the task to react as soon and as accurate as possible. The order of the 144 trials was randomized for each gymnast. There was neither feedback given on the correctness of each trial, nor on the choice reaction time of each trial. The third phase of the experiment took place immediately after the mental rotation task was completed. First, a manipulation check was conducted in which the gymnast was asked for his assumptions on the specific purposes of the experiment. The manipulation check indicated that gymnasts still remained naïve about the specific purpose of the experiment. After the manipulation check, the gymnast was told the specific purposes of the experiment and received a chocolate bar as a reward for participation.

\section{Data Analysis}

A significance criterion of $\alpha=5 \%$ was defined for all results reported. Prior to testing the main hypothesis, possible moderating effects of age were assessed. There was no significant moderating effect of age on the dependent variable. A univariate analysis of variance (ANOVA) with repeated measures was conducted in order to assess differences in choice reaction times between experimental conditions (Tabachnick \& Fidell, 2007). "Gymnasts' Rotation Preference" (anti-clockwise vs. clockwise) was treated as a between-subject factor, whereas "Rotation Direction of Reference Figure" (anti-clockwise vs. clockwise), "Arm Position of Figures" (symmetrical vs. asymmetrical), and "Angular Rotation" $\left(0^{\circ}, 60^{\circ}, 120^{\circ}, 180^{\circ}, 240^{\circ}, 300^{\circ}\right)$ were treated as within-subject factors. When the sphericity assumption was violated, the Greenhouse-Geisser correction was calculated and the degrees of freedom were adjusted. Cohens' $f$ was calculated as an effect size for all $F$-values reported (Cohen, 1988).

\section{Results of Research}

It was expected that gymnasts who prefer a clockwise rotation direction in a forward twisting salto show faster reaction times on rotation-preference congruent stimuli in a mental rotation test as compared to rotation-preference incongruent stimuli. Since gymnasts performed a psychometric mental rotation test, it was also expected that mental rotation performance in general varies as a function of the angular rotation between the two stimuli figures.

First, results confirmed a main effect of Angular Rotation on choice reaction times, $F(3.53$, $109.43)=19.16, p<0.01$, Cohens' $f=0.79$. Choice reaction times differed clearly as a function of Angular Rotation between the two stimuli figures (see Figure 2). There was an additional main effect of Arm Position of Stimuli Figures on gymnasts' reaction times, $F(1,31)=48.42, p<0.01$, Cohens' $f$ $=1.24$, indicating that gymnasts exhibited slightly faster reaction times when stimuli figures showed symmetrical arm positions as compared to when stimuli figures showed asymmetrical arm positions (mean \pm standard error: $1826 \pm 89 \mathrm{~ms}$ vs. $2327 \pm 179 \mathrm{~ms}$ ). 


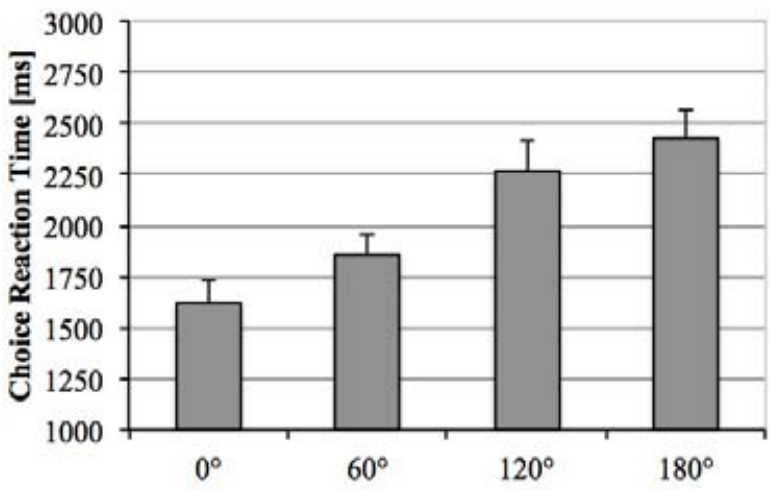

Figure 2: Illustration of gymnasts' choice reaction times as a function of Angular Rotation between the two stimuli figures. Note: Error bars represent standard errors. $60^{\circ}$ and $240^{\circ}$ Angular Rotation as well as $120^{\circ}$ and $300^{\circ}$ Angular Rotation were averaged since there were no significant differences.

Second and consistent with our main hypothesis, there was a significant interaction effect of Rotation Direction of Reference Figure $\times$ Gymnasts' Rotation Preference, $F(1,31)=4.77, p=0.04$, Cohens' $f=0.39$. Gymnasts' who preferred a clockwise rotation preference showed faster choice reaction times when the rotation direction of the reference figure was clockwise and vice versa (see Figure 3).

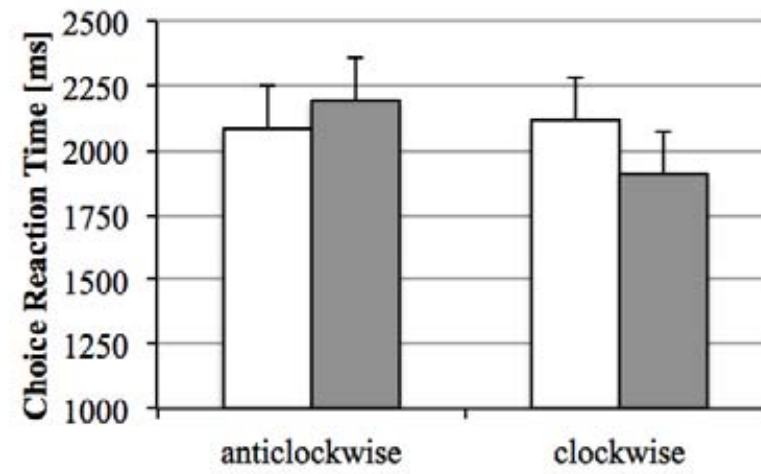

$\square$ anticlockwise rotation preference

$\square_{\text {clockwise rotation }}$ preference

Rotation Direction of Reference Figure

\section{Figure 3: I llustration of gymnasts' choice reaction times as a function of Rotation Direction of Reference Figure and gymnasts' Rotation Preference. Note: Error bars represent standard errors.}

Results furthermore revealed two additional significant interaction effects as a consequence of the main and interaction effects just mentioned: Rotation Direction of Reference Figure $\times$ Angular Rotation $\times$ Gymnasts' Rotation Preference, $F(5,155)=2.70, p=0.02$, Cohens' $f=0.30$, and Rotation Direction of Reference Figure $\times$ Arm Position of Stimuli Figures $\times$ Angular Rotation, $F$ (3.67, $113.81)=3.85, p<0.01$, Cohens' $f=0.35$. There were neither further significant main, nor interaction effects of the experimental factors on gymnasts' choice reaction time.

\section{Discussion}

The purpose of this study was to investigate the role of specific sensory-motor experience on mental rotation performance in gymnasts. It was predicted that the gymnast's mental rotation perfor- 
mance on gymnast-like stimuli varies as a function of gymnasts' rotation preference when performing forward twisting saltos. In particular, it was expected that gymnasts who prefer a clockwise rotation direction in a forward twisting salto show faster reaction times on rotation-preference congruent stimuli in a mental rotation test as compared to rotation-preference incongruent stimuli. Since gymnasts were asked to perform a mental rotation test, it was also expected that mental rotation performance in general varies as a function of the angular rotation between the two stimuli figures.

First, the results revealed that choice reaction times differed clearly as a function of Angular Rotation between the two stimuli figures, thus replicating for instance Shepard and Metzlers' (1971) pattern of results and arguing in favor of the functional equivalence hypothesis. The functional equivalence between percepts and represented physical phenomena seems to be directly grounded in the mechanical properties of flying and rotating rigid bodies. Given that a rigid body possesses a constant angular momentum when airborne, there is a systematic relationship between the angular rotation of that body and the time it takes to achieve this rotation. This is especially the case when there are no changes in the moment of inertia, as it was the case in the stimuli used in the mental rotation test.

In line with our main hypothesis, results furthermore revealed that gymnasts who preferred a clockwise rotation preference showed faster choice reaction times when the rotation direction of the reference figure was clockwise and vice versa. This result clearly supports the notion that mental rotation performance varies as a function of sensory-motor system characteristics between different people. In particular, sensory-motor experience in terms of rotational preference seems to facilitate mental rotation of rotation-preference congruent stimuli. Gymnasts' have developed their rotation preference already early in their training and this preference is usually maintained throughout their whole career (Sands, 2000). Therefore they exhibit substantial sensory-motor experience in one rotation direction about the longitudinal axis, since often this rotation preference generalises to other skills.

There are several limitations of this study and two aspects should be highlighted: First, it was argued that athletes' sensory-motor system usually considerably differs from non-athletes, and thus one would expect that mental rotation performance varies as a function of sensory-motor system characteristics between different people (Enoka, 2003). Our study revealed for instance that gymnasts who prefer a clockwise rotation direction in a forward twisting salto show faster reaction times on rotation-preference congruent in a mental rotation test as compared to rotation-preference incongruent stimuli. The question hereby is, if such relationships also occur for phenomena that exist due to different rotation demands in different sports. For instance, gymnastics and soccer place different (rotation) demands on athletes, which in turn may lead to considerably different sensory-motor experiences over years of practice, and thus to differences in cognitive processing. Second, in this study, gymnasts performed a psychometric mental rotation test whilst sitting in front of a computer monitor. The question is, if concurrent movements such as symmetrical or asymmetrical arm-, leg-, trunk-, or head-movements would facilitate mental rotation performance (e.g., Wohlschläger \& Wohlschläger, 1998). Given that gymnasts use a particular movement of the arms to initiate twists about the longitudinal axis when airborne, one would assume that a concurrent and yet rotationdirection and/or rotation-preference congruent arm movement during mental rotation should facilitate the mental rotation process and vice versa.

There are, however, some practical consequences of this study so far. First, sensory-motor experience in a particular sport may facilitate cognitive processing of specific stimuli. This may be advantageous for situations in which people are engaged in observing sport performance (officials, judges, coaches). This may especially be helpful when the observer has to make decision under timeconstraints. Second and seen the other way round, motor learning processes could potentially benefit from particular mental rotation tasks (or simulations) integrated into physical training. Assuming that a ,mental rotation training' may also lead to a better development of mental representations of particular rotation skills, it may be beneficial to prevent problems such as orientation loss which can be seen as a particular problem in artistic sports (Day, Thatcher, Greenlees, \& Woods, 2006). 


\section{Conclusions}

This study examined mental rotation performance in gymnasts with either clockwise or anticlockwise rotation preference. Results revealed that choice reaction times differed clearly as a function of Angular Rotation between the two stimuli figures, and that gymnasts who preferred a clockwise rotation preference showed faster choice reaction times when the rotation direction of the reference figure was clockwise and vice versa. This result clearly supports the notion that mental rotation performance varies as a function of sensory-motor system characteristics between different people. The congruence between stimuli and participants' sensory-motor experiences seems to be a significant predictor of mental rotation performance in athletes. On a transfer level, this argument would at least in part change practitioners' understanding of motor learning. It could furthermore contribute to the training of athletes from sports such as sky-diving, scuba-diving, and climbing, where losses of spatial orientation can be life-threatening.

\section{References}

Amorim, M. A., Isableu, B., \& Jarraya, M. (2006). Embodied spatial transformations: "Body Analogy" for the mental rotation of objects. Journal of Experimental Psychology: General, 135, 327-347.

Barsalou, L. W. (2008). Grounded cognition. Annual Review of Psychology, 59, 617-645.

Blake, R., \& Shiffrar, M. (2007). Perception of human motion. Annual Review of Psychology, 58, 47-73.

Cohen J. (1988). Statistical power analysis for the behavioural sciences (2nd Ed.). Hillsdale, NJ: Erlbaum.

Day, M. C., Thatcher, J., Greenlees, I., \& Woods, B. (2006). The causes of psychological responses to lost move syndrome in national level trampolinists. Journal of Applied Sport Psychology, 18, 151-166.

E-Frontier (2007). Poser 7 Mac/Win. Watsonville: Curious Labs.

Enoka, R. M. (2002). Neuromechanics of human movement (3rd Ed.). Champaign, IL: Human Kinetics.

Gibbs, R. W. (2006). Embodiment and cognitive science. New York, NY: Cambridge University Press.

Heinen, T. (2010). Cognition and Movement Chronometry (CMC) - Software. Cologne: German Sport University Cologne.

Heinen, T. (2013). Does the athletes' body shape the athletes' mind? A few ideas on athletes' mental rotation performance. Commentary on Jansen and Lehmann. Advances in Cognitive Psychology, 9 (2), 99-101.

Ionta, S., Fourkas, A. D., Fiorio, M., \& Aglioti, S. M. (2007). The influence of hands posture on mental rotation of hands and feet. Experimental Brain Research, 183, 1-7.

Jansen, P., \& Lehmann, J. (2013). Mental rotation performance in soccer players and gymnasts in an objectbased mental rotation task. Advances in Cognitive Psychology, 2, 92-98.

Jolicoeur, P., \& Kosslyn, S. (1985). Demand characteristics in image scanning experiments. Journal of Mental Imagery, 9 (2), 41-49.

Lenggenhager, B., Lopez, C., \& Blanke, O. (2008). Influence of galvanic vestibular stimulation on egocentric and object-based mental transformations. Experimental Brain Research, 184, 211-221.

Moreau, D., Clerc, J., Mansy-Dannay, A., \& Guerrien, A. (2012). Enhancing spatial ability through sport practice: evidence for an effect of motor training on mental rotation performance. Journal of Individual Differences, 33 (2), 83-88.

Munzert, J., Lorey, B., \& Zentgraf, K. (2009). Cognitive motor processes: the role of motor imagery in the study of motor representations. Brain Research Reviews, 60 (2), 306-326.

O’Regan, J. K., \& Noë, A., (2001). A sensorimotor account of vision and visual consciousness. Behavioral and Brain Sciences, 24, 939-1031.

Paivio, A. (1990). Mental representations. A dual coding approach. Oxford: Oxford University Press Inc.

Pietsch, S., \& Jansen, P. (2012). Different mental rotation performance in students of music, sport and education. Learning and Individual Differences, 22 (1), 159-163.

Sands, W. (2000). Twist Direction. Technique, 20 (2), 5-7. 
Shepard, R. N., \& Metzler, J. (1971). Mental rotation of three-dimensional objects. Science, 171 (3972), 701703.

Sternberg, R. J. (2003). Cognitive psychology (3rd Ed.). Belmont, CA: Wadsworth.

Tabachnick, B. G., \& Fidell, L. S. (2007). Using multivariate statistics (5th Ed.). Boston: Allyn \& Bacon.

Wexler, M., Kosslyn, S. M., \& Berthoz, A. (1998). Motor processes in mental rotation. Cognition, 68, 7794.

Wohlschläger, A., \& Wohlschläger, A. (1998). Mental and manual rotation. Journal of Experimental Psychology: Human Perception and Performance, 24, 397-412.

Yeadon, M. R. (1990). The simulation of aerial movement. II. A mathematical inertia model of the human body. Journal of Biomechanics, 23, 67-74.

Yeadon, M. R. (2000). Aerial movement. In V.M. Zatsiorsky (Ed.), Biomechanics in sport: performance enhancement and injury prevention (pp. 273-283). Oxford: Blackwell Science Ltd.

Zacks, J. M. (2008). Neuroimaging studies of mental rotation: a meta-analysis and review. Journal of Cognitive Neuroscience, 20 (1), 1-19.

\section{Advised by Serhiy Boltivets, Grigory Kostyuk Psychological Institute of the Ukrainian National Academy of Pedagogical Sciences, Ukraine}

